\title{
HUMAN - ROBOTS SAFE COOPERATION IN AN INTEGRATED APPROACH
}

\author{
Mugur Spirescu ${ }^{1}$, Sergiu Dumitru ${ }^{2}$, Alexandru Constantinesu ${ }^{3}$, Cristian Badea ${ }^{4}$ \\ 1,2,3,4 National Institute of Research and Development in Mechatronics and Measurement Technique, \\ Bucharest, Romania \\ mugur.spirescu@gmail.com, sergiu.dumitru@incdmtm.ro, alexandru.constantinescu@incdmtm.ro, \\ adresacontact@gmail.com
}

\begin{abstract}
Since Dacia Renault, one of our old customer, decided to increase the number of robots in the production lines, INCDMTM was put in the situation to adapt to this situation. Possible collaboration of humans and robots in shared workplaces opens up new possibilities and concepts in industry and production. The standardisation and legal situation allows for human-robot collaboration (HRC) within defined limits. In all cases involving HRC, protection of the human must be ensured by safety measures. The technology used must meet certain safety requirements. According to the risk assessment the essential safety and health requirements are determined and corresponding measures are taken. Our engineers designed and built a system which is reproducing the working conditions for a safe interaction between robots and humans in a shared workspace
\end{abstract}

Keywords: Robotics Safety, Human-Robot Interaction, Safety Standards, Safety Devices.

\section{Introduction}

Until recent decades, however, the history of robots was more science fiction than reality. Even after the1960s, when the first industrial robots moved onto America's automobile assembly lines, technical advances and applications lagged behind futurists' predictions.

Today, major technological advances -including the microprocessor, artificial intelligence techniques, and innovations in automation and control systems -have ushered in a new age of robotics, in which once-futuristic visions have either become realities or are on the horizon. Industrial robots are programmable multifunctional mechanical devices designed to move material, parts, or specialized devices programmed to perform a variety of tasks.

An industrial robot system includes not only industrial robots but also any devices and/or sensors needed to perform their tasks and sequencing and monitoring interfaces for communication. Robots are generally used to perform tasks unsafe, dangerous, highly repetitive and unpleasant. They have many different functions, such as material handling, assembly, welding and functions of loading/unloading machine tools, painting, spraying, etc.
Most robots are designed for an operation by the technique teach - and - repeat.

In this way, a trained operator (programmer) typically uses a portable control to learn a robot about its task. The speed of the robot during these sessions are slow.

\section{Accidents}

Studies in Sweden and Japan indicate that many robot accidents do not take place under normal operating conditions, but in programming, adjustment or optimization, maintenance, repair, testing, installation.

During many of these operations, the operator, scheduler or maintenance worker may be temporarily in the robot workspace, in which case unintended operations could lead to accidents.

Typical accidents include the following:

- robot arm operated erratically during programming sequence and hit the operator.

- the operator of a robot manipulator of material entered the workspace during operations and was caught between the rear of the robot and a safety zone.

- a colleague accidentally operated power switch, while a maintenance worker is working on robot. Robot arm hit the hand of the maintenance worker. 


\section{Robotics Security}

Proper selection of an effective robotics security system should be based on a risk analysis of operations in use, programming and maintenance of therobot.

Among the elements to be analyzed are tasks for which a robot will be programmed, start-up command or programming procedures, environmental conditions, requirements and installation location, possible human error, scheduled maintenance and unscheduled possible malfunctioning robot - system, normal operation, and all staff functions and tasks.

An effective security system protects not only operators but also engineers, programmers, maintenance staff and other persons working on or with the machine and could be exposed to the hazards associated with the operation of the robot. A combination of methods can be used to protect. Backup systems are recommended particularly if a robot or robot system operate in hazardous conditions or handling hazardous materials. Protective devices used should not represent or act as a threat.

Sources of danger. The estimated human hazards can be associated with several additional variants as follows.

- Human errors. Due to the timing prior activation, connecting peripheral equipment inputoutput sensors to the microprocessor or a peripheral can cause dangerous movements, unexpected or uncontrolled actions of the robot. Enabling incorrect remote control or the control panel is a common human error. The biggest problem, however, is the individual himself placed in hazardous locations while the robot is in programming or maintenance is carried out by.

- Errors in control. Intrinsic defects in the control system of the robot, the software error, electromagnetic interference and radio frequency interference control errors. In addition, these errors can occur due to faults hydraulic, pneumatic or electric subsystems associated with the robot.

- Unauthorized access. Entering the robot security area is dangerous because the person concerned may not be familiar with security features or their activation status.

- Mechanical failures. Schedules do not account for accumulated mechanical part errors and unexpected malfunctions may occur.

- Environmental sources. Electromagnetic and radio frequency interference (transients signals) should be considered to exert undue influence on the operation and increase the potential for injury to anyone working in the area. Solutions to environmental risks must be documented before start up.
- Supply system. Power sources, pneumatic, hydraulic or electrical control malfunction or that the transmission elements in the power system can disrupt electrical control signals and / or power lines. The risk of fire is increased electrical overload or use of flammable hydraulic oil. Electric shock and release energy storage devices also can be hazardous to personnel.

- Improper installations. Design requirements and arrangement of equipment, utilities, facilities, if done inappropriately can lead to risks.

\section{Safety Standards}

The development of guidelines and requirements in the form of international safety standards represents an important effort toward ensuring safety during human-robot interaction.

The International Organization for Standardization (ISO) has been working toward releasing documents that specify how best to maintain safety during interaction between humans and industrial robots. The first step in this process was the release of the ISO 10218 document entitled "Robots and robotic devices - Safety requirements for industrial robots," which is composed of two parts: "Robots" and "Robot systems and integration".

The ISO 10218 outlines some potential methods of safe collaborative manipulation - for example, speed and separation monitoring and power and force limiting - as well as relevant safety requirements.

The technical specification accompanying this document is the ISO/TS 15066 (entitled "Robots and robotic devices - Collaborative robots")

This technical specification provides additional information and details about how to achieve the requirements established by ISO 10218. It includes quantitative biomechanical limits, such as allowable peak forces or pressures for various parts of the body, as well as equations for speed and separation monitoring.

In support of the development of the ISO technical specification, organizations including the National Institute of Standards and Technology (NIST) collaborated with ISO to develop protocols and metrics that would allow for characterization of the effectiveness of a robot's safety methods. While the development of the aforementioned international safety standards represents a crucial first step toward improving HRI (Human Robot Interaction) safety, it is important to note that these standards are being developed specifically for industrial applications.

Although many of the principles would likely transfer to other types of robots and applications, the standards' scope is too narrow to fully address other uses, such as robotic tour guides or assistants for the elderly. 
We therefore must look beyond these industrial standards in order to identify all the pertinent aspects of safe HRI and the various possible safety methods that could be employed to address them.

\section{Our System}

If the risk assessment shows that a machine or process carries a risk of injury, the danger must be removed or stopped.

How to do this depends on the nature and danger of the machine. Safeguards are defined as methods that prevent access to a hazard or detect access to a hazard.

Safeguards include devices like fixed fences, fences interlocked, light curtains, safety mats, twohand controls and switches uride validation. The system built by INCDMTM consists of devices that comprise a significant portion of the above and take into account the specific conditions of the robot served.

These devices have been used depending on the intended purpose and there are as follows:

- Fixed fences

If the risk is part of the equipment that does not require access, a fence should be permanently fixed to the machine. These types of fences must require tools for removal. Fixed fences must be able to 1) withstand their operating environment, 2) contain protection where necessary, and 3) not create hazards by having, for example, sharp edges. Fixed fences may have openings where the guard used equipment or openings due to the use of a wire mesh enclosures. They are supplied by Satech Safety Technology Spa.

- Access detecting

Protection is used to detect access to a hazard. When detection is selected as the method of risk reduction, the designer must understand that they must be used in a complete safety; protection device, by itself, does not provide risk reduction. This safety system generally consists of three blocks: 1) an input device that detects access to danger, 2) a logic device that processes signals from the sensing device, checks the status of the security system, and then starts or stops the output devices and 3) an output device that controls the actuator (for example, a motor).

- Detection means

Many alternative devices are available to detect the presence of a person entering or inside a hazard area. The best choice for an application depends on a number of factors.

$>$ access frequency,

$>$ time off danger

$>$ importance of completing the machine cycle, and

$>$ protection from projectiles, fluids, mists, vapors, etc.
Presence detection devices such as light curtains, safety mats and scanners, provide quick and easy access to the danger zone and are often selected when operators must frequently access the hazard area.

The best choice of protective measure is a device or system that provides maximum protection with minimum obstruction to the normal operation of the machine. Must be taken into account all aspects of using the machine, as experience shows that a system is difficult to use is more likely to be removed or bypassed.

- Safety light curtains (Wenglor SG4-30IE060C1 / SG4-30IS060C1)

Safety light curtains are most simply described as photoelectric presence sensors specifically designed to protect personnel from injuries related to hazardous machine movement. They are ideal for applications where personnel need frequent and easy access to an operating point of highly hazardous potential.

- Switches key lock (PILZ PSENCS2. 1P / PSENCS2. 1)

Key locking devices requires a drive in the form of language to be inserted and removed. When the key is inserted, the internal safety contacts close and allow the device to work. When the key is removed, the internal safety contacts open and send a stop command to the safety related parts of the control system.

- Emergency Stop function (Schneider Electric XALK178G)

Emergency stop function must operate as either a category 0 or category 1 stop, as determined by a risk assessment. It must be initiated by a single human action. When executed, it must override all other functions and machine operating modes. The objective is to remove power as quickly as possible, without creating additional risks.

- Programmable Logic Controllers (Siemens S7-300)

The need for flexible security applications led to the development of safety PLCs controllers. Programmable safety controllers provide users the same level of control flexibility in a safety application that they are accustomed standard. Today, both computers and embedded microprocessors are used to control all industrial robots. They perform all necessary computational functions and interface control sensors associated with grippers, tools and other related peripherals.

There are a lot of other devices wich can be used for increasing the safety in working with robots. Here are some of the projects developed recently by the researchers.

The results of these projects will be take into consideration by the engineers from INCDMTM for further development of our activity implying the work with the robots. 


\section{Future Directions}

Development and Validation of Tactile Sensor Systems for Reliable Collision Detection during Human-Robot Interaction.

There will be great need in the future for robotic systems that share work areas with humans or work directly with them. Such human-robot collaboration is intended to combine humans' cognitive and highly flexible capabilities with robots' strengths such as precision, load carrying capacity and indefatigability.

The prerequisite for human-robot cooperation is human safety. A human may not be injured in the event of a collision. The Fraunhofer IFF in Magdeburg is developing a groundbreaking key technology for human-robot collaboration, a tactile, pressure-sensitive sensor system that reliably detects a robot's collisions with humans and objects and stops it immediately in the event of a collision. The sensor system's distinctive features are its inexpensive sensor configuration, high robustness and customizability for complex robot surfaces. It will be usable on any type of robot. In this project, the Fraunhofer IFF is studying and validating the complex requirements and correlations of materials science, manufacturing, electronics and software.

\section{Safe Person Detection}

Tactile sensor systems integrated in flooring can measure the forces acting on it with a spatial resolution. This kind of flooring detects both stationary and moving objects and can be used to make machines and systems safe, or even to track flows of items and people. Pressure-sensitive flooring can additionally be used in the medical and rehabilitation sectors for fall detection and gait analysis. In the project, the workspace of an $\mathrm{ABB}$ IRB1600 industrial robot was completely secured by using pressure-sensitive flooring.

The tactile sensor technology of the Fraunhofer IFF was integrated in standard industrial flooring, and the pressure-sensitive flooring was configured to safely detect and localize persons in the robot's vicinity. Furthermore, its spatially-resolved position detection allowed for the individual warning and safe areas to be freely and dynamically defined. Intrusion into these safe areas is reliably detected, and the robot reacts accordingly, either by slowing down or stopping completely.

The project verified that pressure-sensitive flooring can be used to replace conventional protective barriers. Pressure-sensitive flooring is especially useful in dirty environments, where, for instance, high dust concentrations could preclude the use of optical sensor systems. Humans and robots can co-operate more closely, and the setup and changeover times of manufacturing workplaces are reduced drastically through the use of pressuresensitive flooring.

\section{Experimental Evaluation of Advanced Sensor- Based Supervision and Work Cell Integration Strategies}

In this project, the partners KUKA and Fraunhofer IFF aim at demonstrating safe interaction between robots and humans in a shared workspace environment. This is to be accomplished by using one of the most current robots, the KUKA LWR 4+, aiming at human-robot collaboration scenarios as well as newly developed sensor systems for flexible workspace surveillance.

The project focus is on exploring necessary communication and interaction between the various work-cell components in order to achieve a reasonable safe process.

Therefore, a main achievement is the development of a concept for integration of a sensorsystem and the KUKA LWR, which is based on an analysis and discussion of available communication interfaces concerning the individual communication tasks in possible use cases.

For the generation and establishment of safety spaces we use a novel safety system that is based on conventional camera and projector technics and which was recently developed at the Fraunhofer Institute for Factory Operation and Automation IFF. While safety space boundaries are directly projected onto surfaces present in the environment, a violation of these safety spaces is detected by the monitoring cameras if an object disrupts the emitted light rays.

An important objective of the experiment is the building of an industry-related collaborative workspace by integration of safety system, robot and required hardware accordingly. Further main tasks of the experiment comprise on the one hand the development of planning algorithms used to generate appropriate safety spaces, and on the other hand the adaption of collision algorithms used to detect intrusions of the established safety spaces. Here, the focus is particularly on generation and exchange of robot models annotated by joint angles and velocities, and planned trajectories between subsystems.

\section{Conclusions}

The large body of work suggests a substantial research effort related to safety during human-robot interaction. Interestingly, safety is not always explicitly mentioned as an application of these works; nonetheless, the inclusion of research relevant to safety in HRI across such a large variety of works highlights the importance of this topic. 
Safety in HRI remains an open problem, however, as many of the mentioned sub-fields are still relatively young. The directions on wich the researchers have to focuse could be the following:

- Expanding Post-Collision Control Methods

- CablelessTeachPendants

- Extending Safe Motion Planning

- Human-RobotCollaboration

- Improving Safety Through Prediction

- Robot-to-Robot Synchronization

- Integration of Safety Methods

- Vision-based Guard Systems

\section{References}

[1] https://www.iff.fraunhofer.de/en/businessunits/robotic-systems/taksens.html; accesed at 07.2017

[2] https://www.iff.fraunhofer.de/en/businessunits/robotic-systems/tactile-sensor-systemspressure-sensitive-flooring.html; accesed at 07.2017

[3] https://www.iff.fraunhofer.de/en/businessunits/robotic-systems/echordexecell.html;accesed at 06.2017
[4] EN ISO 10218 "Robots and robotic devices Safety requirements for industrial robots", Parts 1 and 2 ISO 11161"Safety of Integrated Manufacturing Systems - Basic Requirements"

[5] ISO 13849 (EN 954) "Safety related parts of control systems"-Pt 1: General principles for design. Pt 2: Validation

[6] ISO 13850 (EN 418) "Emergency Stop devices, functional aspects-Principles for design"

[7] ISO 13854 (EN 349) "Minimum distances to avoid crushing parts of the human body"

[8] ISO 14119 (EN 1088) "Interlocking devices associated with guards-Principles for design and selection"

[9] ISO 14120 (EN 953) "General Requirements for the Design and Construction of Guards"

[10] ISO/TS 15066 - Safety of collaborative robots

[11] P. A. Lasota, T. Fong and J. A. Shah - A Survey of Methods for Safe Human-Robot Interaction Foundations and Trends R in Robotics Vol. 5, No. 4 (2014)

[12] G. Schuster, M. Winrich- SAFETY-WP009A-ENP-December2009 